\title{
Correction to: Inflation with $F(T)$ teleparallel gravity
}

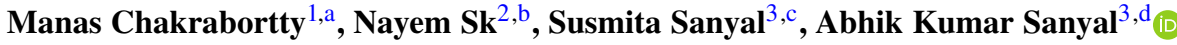 \\ ${ }^{1}$ Department of Physics, Bankura University, Bankura 722155, India \\ 2 Department of Physics, Saidabad Manindra Chandra Vidyapith, Murshidabad 742103, India \\ 3 Department of Physics, Jangipur College, Murshidabad 742213, India
}

(C) The Author(s), under exclusive licence to Società Italiana di Fisica and Springer-Verlag GmbH Germany, part of Springer Nature 2021

\section{Correction to: Eur. Phys. J. Plus (2021) 136:1213 https://doi.org/10.1140/epjp/s13360-021-02232-y}

In this article, the following equations have been adjusted:

On page $4, n_{s}<0.5$ and $n_{s}<0.1$ have been changed to $n_{s} \sim 0.5$ and $n_{s} \sim 0.1$, respectively.

$a(t)=a_{0} e A^{(\ln t) \lambda}$ has been adjusted to $a(t)=a_{0} e A^{(\ln t) \lambda}$.

On page $9,46 \leq N \leq 60$ has been placed in parentheses, $(46 \leq N \leq 6)$.

$\gamma V_{1}=1 M_{P}^{5}$ has been adjusted to $\gamma V_{1}=1 M_{P}^{5}$.

The original article has been corrected.

The original article can be found online at https://doi.org/10.1140/epjp/s13360-021-02232-y.

\footnotetext{
a e-mail: manas.chakrabortty001@gmail.com

b e-mail: nayemsk1981@gmail.com

c e-mail: susmitasanyal@yahoo.com

de-mail: sanyal_ak@yahoo.com (corresponding author)
} 